\title{
Self-Dual and Characterization of Partial Dual Graphs
}

\author{
S.Lakshmi ${ }^{1}$ N.Saranya ${ }^{2}$ \\ ${ }^{I}$ Department of Mathematics, PSGR Krishnammal College for Women, Coimbatore 641004, Tamil Nadu, India \\ ${ }^{2}$ Department of Mathematics, PSGR Krishnammal College for Women, Coimbatore 641004, Tamil Nadu, India
}

\begin{abstract}
In this paper we consider the three forms of self-duality that can be exhibited by a planar graph $G$, map self-duality, graph self-duality, matroid self-duality. We show how these concepts are related with each other and with the connectivity of $G$. Also we go to characterize partial duality of graphs in terms of bijection between edge sets of corresponding graphs. This result generalizes a well known result of J.Edmonds in which natural duality of graphs is characterized in terms of edge correspondence, and gives combinatorial characterization of partial duality.
\end{abstract}

\section{Introduction}

Self-dual graph was developed by Brigitte Servatius and Herman Servatius. The three forms of selfduality that can be exhibited by a planar graph $G$, map self-duality, graph self-duality, matroid self-duality. They shown how these concepts are related with each other and with the connectivity of $G$. We use the geometry of self-dual polyhedra together with the structure of the cycle matroid to construct all self-dual graphs.

S. Chmutov recently introduced the concept of the partial dual $\mathrm{G}^{\mathrm{A}}$ of a ribbon graph $\mathrm{G}([8])$. Partial duality generalizes the natural dual (or Euler-Poincare dual or geometric dual) of a ribbon graph by forming the dual of G only with respect to a subset of its edges A (a formal definition of partial duality is given in Section 3.2). In contrast with natural duality, where the topologies of $G$ and $G^{*}$ are similar, the topology of a partial dual $G^{A}$ can be very different from the topology of $G$. For example, although a ribbon graph and its natural dual always have the same genus, a ribbon graph and a partial dual need not.

As one would expect with a generalization of duality, partial duality has desirable properties. For example, (up to normalization and specialization) the weighted (Bollobas-Riordan) ribbon graph polynomials of $\mathrm{G}$ and $\mathrm{G}^{\mathrm{A}}$ are equal. This generalizes the well known relation between the Tutte polynomial of a plane graph and its natural dual: $T(G ; x ; y)=T\left(G^{*} ; y ; x\right)$.

\section{Self-Dual Graphs}

\subsection{Forms of self-duality} Definition 2.1

Example

A planar graph is isomorphic to its own dual is called a self-dual graph.

$K_{4}$ is a Self-dual graph.

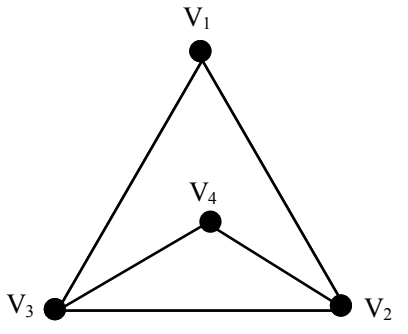

Fig.:2.1

\section{Definition 2.2}

Given a planar graph $\mathrm{G}=(\mathrm{V}, \mathrm{E})$, any regular embedding of the topological realization of $\mathrm{G}$ into a sphere partitions the sphere into regions called the faces of the embedding, and we write the embedded graph, called a map, as $\mathrm{M}=(\mathrm{V}, \mathrm{E}, \mathrm{F})$. $\mathrm{G}$ may have loops and parallel edges.

\section{Definition 2.3}

Given a map $\mathrm{M}$, form the dual map, $\mathrm{M}^{*}$ by placing a vertex $f^{*}$ in the centre of each face $\mathrm{f}$, and for each edge e of $M$ bounding two faces $f_{1}$ and $f_{2}$, draw a dual edge e* connecting the vertices $f_{1}^{*}$ and $f_{2} *$ and crossing e once transversely. Each vertex $\mathrm{V}$ of $\mathrm{M}$ will then correspond to a face $\mathrm{V}^{*}$ of $\mathrm{M}^{*}$ and write $\mathrm{M}^{*}=\left(\mathrm{F}^{*}\right.$, $\left.\mathrm{E}^{*}, \mathrm{~V}^{*}\right)$. If the graph $\mathrm{G}$ has distinguishable embeddings, then $\mathrm{G}$ may have more than one dual graph, see Fig.: 2.2. In this example a portion of the map (V, E, F) is flipped over on a separating set of two vertices to form $\left(\mathrm{V}, \mathrm{E}, \mathrm{F}^{\prime}\right)$. 

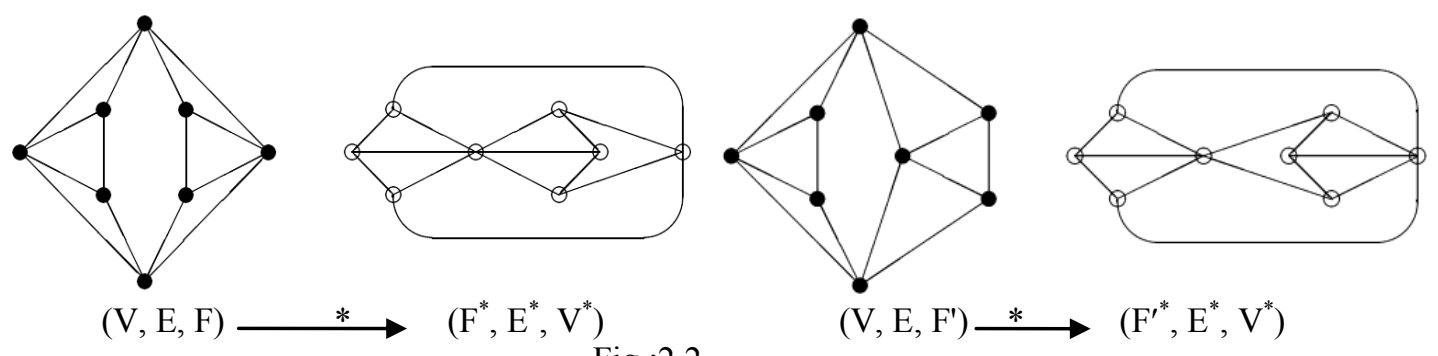

Fig.:2.2

Such a move is called Whitney flip, and the duals of $(\mathrm{V}, \mathrm{E}, \mathrm{F})$ and $\left(\mathrm{V}, \mathrm{E}, \mathrm{F}^{\prime}\right)$ are said to differ by a Whitney twist. If the graph $(\mathrm{V}, \mathrm{E})$ is 3-connected, then there is a unique embedding in the plane and so the dual is determined by the graph alone.

Given a map $X=(V, E, F)$ and its dual $X^{*}=\left(F^{*}, E^{*}, V^{*}\right)$, there are three notions of self-duality. The strongest, map self-duality, requires that $\mathrm{X}$ and $\mathrm{X}^{*}$ are isomorphic as maps, that is, there is an isomorphism $\delta:(\mathrm{V}, \mathrm{E}, \mathrm{F}) \rightarrow\left(\mathrm{F}^{*}, \mathrm{E}^{*}, \mathrm{~V}^{*}\right)$ preserving incidences. A weaker notion requires only a graph isomorphism $\delta:(V, E) \rightarrow\left(F^{*}, E^{*}\right)$, in which case that the map $(V, E, F)$ is graph self-dual, and we say that $G=(V, E)$ is a self-dual graph.

\section{Definition 2.4}

A geometric duality is a bijection $\mathrm{g}: \mathrm{E}(\mathrm{G}) \rightarrow \mathrm{E}\left(\mathrm{G}^{*}\right)$ such that $e \in \mathrm{E}$ is the edge dual to $\mathrm{g}(e) \in \mathrm{E}\left(\mathrm{G}^{*}\right)$. If $M$ is 2-cell, then $M$ is connected so if $M$ is a 2-cell embedding, then $\left(M^{*}\right)^{*} \cong M$ (we use * to indicate the geometric dual operation).

\section{Definition 2.5}

An algebraic duality is a bijection $\mathrm{g}: \mathrm{E}(G) \rightarrow \mathrm{E}(\hat{G})$ such that $\mathrm{P}$ is a circuit of $\mathrm{G}$ if and only if $\mathrm{g}(\mathrm{p})$ is a minimal edge-cut of $\hat{G}$. Given a graph $\mathrm{G}=(\mathrm{V}, \mathrm{E})$, an algebraic dual of $\mathrm{G}$ is a graph $\hat{G}$ for which there exists an algebraic duality $\mathrm{g}: \mathrm{E}(\mathrm{G}) \rightarrow \mathrm{E}(\hat{G})$.

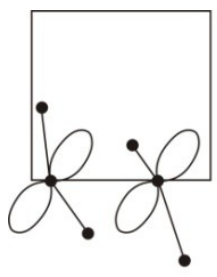

(a)

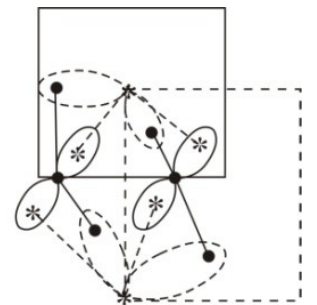

(b)

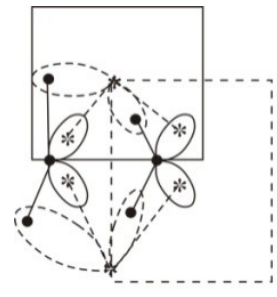

(c)

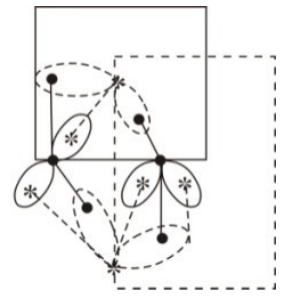

(d)

Fig.:2.3. A graph and several of its embeddings.

The geometric duals are shown in dotted lines. Embedding (b) is map self-dual, (c) is graphically selfdual and (d) is algebraically self-dual. Now define several forms of self-duality. Let $G=(V, E)$ be a graph and let $\mathrm{M}=(\mathrm{V}, \mathrm{E}, \mathrm{F})$ be a fixed map of $\mathrm{G}$, with geometric dual $\mathrm{M}^{*}=\left(\mathrm{F}^{*}, \mathrm{E}^{*}, \mathrm{~V}^{*}\right)$.

\section{Definition 2.6}

1. $M$ is map self-dual if $M \cong M^{*}$.

2. $\mathrm{M}$ is graphically self-dual if $(\mathrm{V}, \mathrm{E}) \cong\left(\mathrm{F}^{*}, \mathrm{E}^{*}\right)$.

3. $\mathrm{G}$ is algebraically self-dual if $\mathrm{G} \cong \mathrm{G}^{*}$, where $\hat{G}$ is some algebraic dual of $\mathrm{G}$.

Theorem 2.1

For $\mathrm{w} \geq 1$, there exists a self-dual embedding of some graph $\mathrm{G}$ of order $\mathrm{n}$ on $S_{n(w-1)+1}$ if and only if $\mathrm{n} \geq 4 \mathrm{w}+1$.

Note that a self-dual graph need not be self-dual on the surface of its genus. A single loop is planar however it has a (non 2-cell) self-dual embedding on the torus.

Also note that there are infinitely many self-dual graphs. One such infinite family for the plane is the wheels. A wheel $W_{n}$ consists of cycle of length $\mathrm{n}$ and a single vertex adjacent to each vertex on the cycle by means of a single edge called a Spoke. The complete graph on four vertices is also $W_{3}$. See Fig.:2.4 for $W_{6}$. 


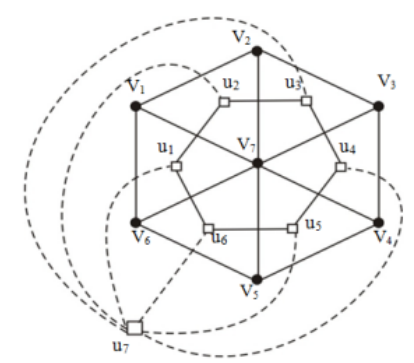

Fig.:2.4.The 6-Wheel and its dual

Matroids

Matroids may be considered a natural generalization of graphs. Thus when discussing a family of graphs, we should also consider the matroidal implications.

\section{Definition 2.7}

Let $\mathrm{S}$ be a finite set, the ground set, and let $\mathrm{I}$ be a set of subsets of $\mathrm{S}$, the independent sets. Then $\mathrm{M}=$ $(S, I)$ is a matroid if:

1. $\phi \in I$;

2. If $J^{\prime} \subseteq J \in I$, then $J^{\prime} \in I$; and

3. For all $\mathrm{A} \subseteq \mathrm{S}$, all maximal independent subsets of $\mathrm{A}$ have the same cardinality.

An isomorphism between two matroids $\mathrm{M}_{1}=\left(S_{1}, I_{1}\right)$ and $\mathrm{M}_{2}=\left(S_{2}, I_{2}\right)$ is a bijection $\chi: S_{1} \rightarrow S_{2}$ such that $\mathrm{I} \in I_{1}$ if and only if $\chi(\mathrm{I}) \in I_{2}$. If such a $\chi$ exists, then $\mathrm{M}_{1}$ and $\mathrm{M}_{2}$ are isomorphic denoted $\mathrm{M}_{1} \cong \mathrm{M}_{2}$

Given a graph $G=(V, E)$, the cycle matroid $M(G)$ of $G$ is the matroid with ground set $E$, and $F \subseteq E$ is independent if and only if $F$ is a forest. A matroid $M$ is graphic if there exists a graph $G$ such that $M=M(G)$.

For a matroid $\mathrm{M}=(\mathrm{S}, \mathrm{I})$ the dual matroid $\mathrm{M}^{*}=\left(\mathrm{S}, \mathrm{I}^{*}\right)$ has ground set $\mathrm{S}$ and $I \subseteq S$ in $\mathrm{I}^{*}$ if there is a maximal independent set $B$ in $M$ such that $I \subseteq S \backslash B$. A matroid $M$ is co-graphic if $M *$ is graphic. It is easily shown that if $G$ is a connected planar graph, then $M *(G)=M\left(G^{*}\right)$. It is well known that $G$ is algebraically self-dual if and only if cycle matroids of $\mathrm{G}$ and $\mathrm{G}^{*}$ are isomorphic.

\subsection{A comparison of self-duality}

It is clear that for a map (V, E, F) we have,

Map self-duality $\Rightarrow$ Graph self-duality $\Rightarrow$ Matroid self-duality.

However, In general, these implications cannot be reversed, as shown by Fig.: 2.3. But, we are concerned to what extent these implications can be reversed.

\subsubsection{Self - Dual Maps and Self - Dual Graphs}

In the previous examples the graphs were of low connectivity, a planar 3-connected simple graph has a unique embedding on the sphere, in the sense that if $\mathrm{p}$ and $\mathrm{q}$ are embeddings, then there is a homeomorphism $\mathrm{h}$ of the sphere so that $\mathrm{p}=\mathrm{hq}$. Any isomorphism between the cycle matroids of a 3 -connected graph is carried by a graph isomorphism. Thus, for a 3-connected graph

Map self-duality $\Leftarrow$ Graph self-duality $\Leftarrow$ Matroid self-duality, So self-dual 3-connected graphs, as well as self-dual 3-connected graphic matroids, reduce to the case of self-dual maps. Since, the examples in Fig.:2.3 are only 1-connected, we must consider the 2-connected case. In Fig.2.5 is an example of a graphically self-dual map whose graph is 2-connected which is not map self-dual. One might hope that, as was the case in Fig.:2.3, that such examples can be corrected by re - embedding or rearranging, however we have the following strong result.

\section{Theorem 2.2.1}

There exists a 2-connected map $(\mathrm{V}, \mathrm{E}, \mathrm{F})$ which is graphically self-dual, so that $(\mathrm{V}, \mathrm{E}) \cong\left(\mathrm{F}^{*}, \mathrm{~V}^{*}\right)$, but for which every map $\left(V^{\prime}, E^{\prime}, F^{\prime}\right)$ such that $\mathrm{M}(\mathrm{E}) \cong \mathrm{M}\left(E^{\prime}\right)$ is not map self-dual.

\section{Proof}

Consider the map in Fig.:2.5 which is drawn on an unfolded cube. The graph is obtained by gluing two 3 -connected self-dual maps together along an edge $(a, b)$ and erasing the common edge. One map has only two reflections as self-dualities, both fixing the glued edge the other has only two rotations of order four as dualities, 
again fixing the glued edge. The graph self-duality is therefore a combination of both, an order 4 rotation followed by a Whitney twist of the reflective hemisphere. It is easy to see that all the embeddings of this graph, as well as the graph obtained after the Whitney flip have the same property.

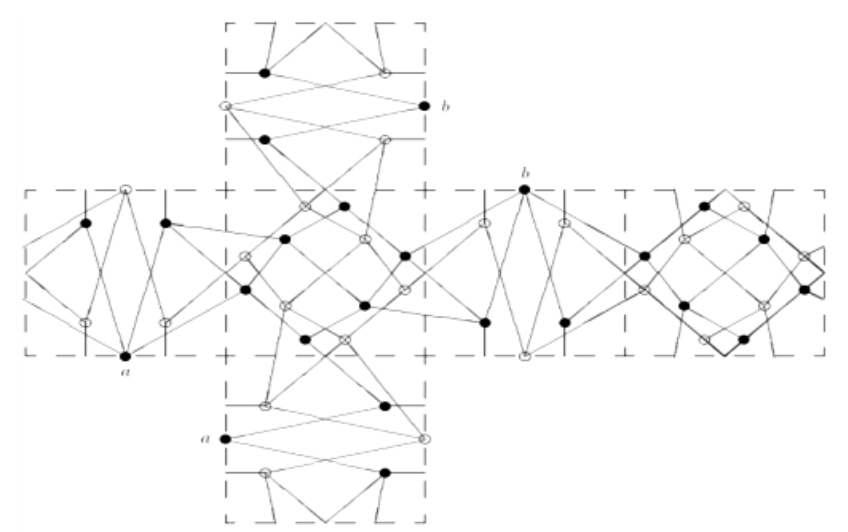

Theorem 2.2.2

Fig: 2.5

There is a graphically self-dual map (V, E, F) with (V, E) 1-connected and having only 3-connected blocks, but for which every map $\left(V^{\prime}, E^{\prime}, F^{\prime}\right)$ such that $\mathrm{M}(E) \cong \mathrm{M}\left(E^{\prime}\right)$ is not map self- dual.

Proof

Consider the 3-connected self-dual maps in Fig.:2.2. $X_{1}$ has only self-dualities of order 4, two rotations and two flip rotations, while $X_{2}$ has only a left-right reflection and a $180^{\circ}$ rotation as a self-duality. Form a new map $\mathrm{X}$ by gluing two copies of $X_{2}$ to $X_{1}$ in the quadrilateral marked with q's, with the gluing at the vertices marked $\mathrm{v}$ and $\mathrm{v}^{*} . \mathrm{X}$ is graphically self-dual, as can easily be checked, but no gluing of two copies of $X_{2}$ can give map self-duality since every quadrilateral in $X_{1}$ has order 4 under any self-duality.

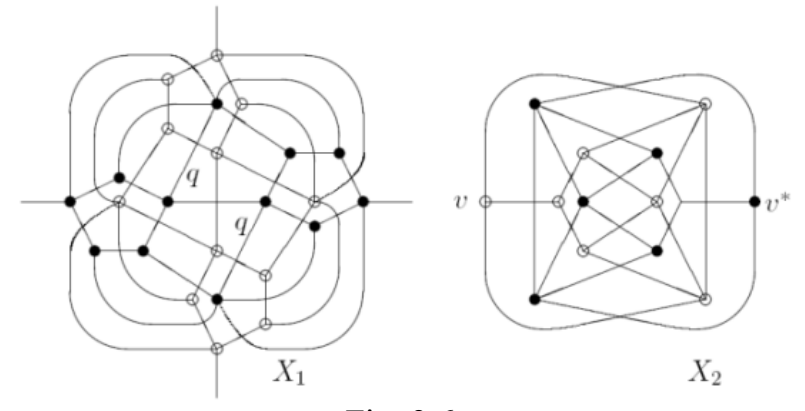

Fig.:2.6

In particular, self-dual graphs of connectivity less than 3 cannot in general be re-embedded as self-dual maps.

\subsubsection{Self-dual graphs and matroids}

If $\mathrm{G}$ is 1-connected, then its cycle matroid has a unique decomposition as the direct sum of connected graphic matroids, $M(\mathrm{G})=M_{1} \oplus M_{2} \oplus \cdots \ldots \oplus M_{k}$, and if $\mathrm{G}^{*}$ is a planar dual of $\mathrm{G}$, then $\mathrm{M}\left(\mathrm{G}^{*}\right)=\mathrm{M}(\mathrm{G})^{*}=$ $M_{1} * \oplus M_{2} * \oplus \cdots \cdots \oplus M_{k} *$. If $\mathrm{G}$ is a graph self-dual, then there is a bijection $\delta: \mathrm{M}(\mathrm{G}) \rightarrow \mathrm{M}\left(\mathrm{G}^{*}\right)$ sending cycles to cycles, and so there is a partition $\pi$ of $\{1,2, \ldots \ldots . . \mathrm{k}\}$ such that $\delta: M_{i} \rightarrow M_{\pi(i)}$, and we that $\mathrm{M}(\mathrm{G})$ is the direct sum of self-dual connected matroids, together with some pairs of terms consisting of a connected matroid and its dual.

\section{Theorem 2.2.3}

Next theorem that not every self-dual matroid arises from a self-dual graph.

There exists a self-dual graphic matroid $\mathrm{M}$ such that for any graph $\mathrm{G}=(\mathrm{V}, \mathrm{E})$ with $\mathrm{M}(\mathrm{G})=\mathrm{M}$, and any embedding $(\mathrm{V}, \mathrm{E}, \mathrm{F})$ of $\mathrm{G},(\mathrm{V}, \mathrm{E}) \not{Z}\left(\mathrm{~F}^{*}, \mathrm{E}^{*}\right)$.

Proof

Consider $M_{1}$ and $M_{2}$, the cycle matroids of two distinct 3-connected self-dual maps $X_{1}$ and $X_{2}$ whose only self-dualities are the antipodal map. 
The matroid $M_{1} \oplus M_{2}$ is self-dual, but its only map realizations are as the 1-vertex union of $X_{1}$ and $X_{2}$, which cannot be self-dual since the cut vertex cannot simultaneously be sent to both "antipodal" faces.

So for 1-connected graphs, the three notions of self-duality are all distinct. For 2-connected graphs, however we have the following.

\section{Theorem 2.2.4}

If $\mathrm{G}=(\mathrm{V}, \mathrm{E})$ is a planar 2-connected graph such that $\mathrm{M}(\mathrm{E}) \cong \mathrm{M}(\mathrm{E})^{*}$, then $\mathrm{G}$ has an embedding $(\mathrm{V}, \mathrm{E}, \mathrm{F})$ such that $(\mathrm{V}, \mathrm{E}) \cong\left(\mathrm{F}^{*}, \mathrm{E}^{*}\right)$.

\section{Proof}

Let (V, E, F) be any embedding of G. Then $\mathrm{G}$ is 2-isomorphic, in the sense of [10] to $\left(\mathrm{F}^{*}, \mathrm{E}^{*}\right)$, and thus there is a sequence of Whitney flips which transform

$\left(\mathrm{F}^{*}, \mathrm{E}^{*}, \mathrm{~V}^{*}\right)$ into an isomorphic copy of $\mathrm{G}$ and act as re-embeddings of $\mathrm{G}$. Thus the result is a new embedding $\left(V, E, F^{\prime}\right)$ of $\mathrm{G}$ such that $(\mathrm{V}, \mathrm{E}, \mathrm{F}) \cong\left(F^{*}, E^{*}, V^{*}\right)$.

Thus, to describe 2-connected self-dual graphs it is enough up to embedding, to describe self-dual 2-connected graphic matroid.

\subsection{Self - Dual matroids Definition}

A polyhedron $\mathrm{P}$ is said to be self-dual if there is an isomorphism $\delta: \mathrm{P} \rightarrow \mathrm{P}^{*}$, where $\mathrm{P}^{*}$ denotes the dual of $\mathrm{P}$. We may regard $\delta$ as a permutation of the elements of $\mathrm{P}$ which sends vertices to faces and vice versa, preserving incidence.

As noted earlier 3-connected self-dual graphic matroids are classified via self-dual polyhedra. On the other hand, 1-connected self-dual matroids are easily understood via the direct sum. Also we show how a 2-connected self-dual matroid $\mathrm{M}$ with self-duality $\delta$ arises via 3 -connected graphic matroids by recursively constructing its 3 -block tree $\mathrm{T}(\mathrm{M})$ by adding orbits of pendant nodes.

\section{Theorem 2.3.1}

Let $\mathrm{M}$ be a self-dual connected matroid with 3-block tree $\mathrm{T}$. Let $T^{\prime}$ be the tree obtained from $\mathrm{T}$ by deleting all the pendant nodes, and let $M^{\prime}$ be the 2-connected matroid determined by $T^{\prime}$. Then $M^{\prime}$ is also selfdual.

\section{Proof}

Let $\mathrm{M}$ be a self-dual connected matroid on a set $\mathrm{E}$, so there is a matroid isomorphism $\Delta: \mathrm{M} \rightarrow \mathrm{M}^{*}$, so $\delta$ is a permutation of $\mathrm{E}$ sending cycles to co-cycles. The 3-block tree of $\mathrm{M}^{*}$ is obtained from that of $\mathrm{M}$ by replacing every label with the dual label, so $\Delta$ corresponds to a bijection $\left(\delta,\left\{\delta_{\alpha}\right\}\right)$ of T onto itself, such that for each node $\alpha$ of T, $\delta_{\alpha}: M_{\alpha} \rightarrow M_{f(\alpha)}$ sends cycles of $M_{\alpha}$ to co-cycles of $M_{f(\alpha)}$. The restriction of $\left(\delta,\left\{\delta_{\alpha}\right\}\right)$ to $T^{\prime}$ has the same property and so corresponds to a self-dual permutation of $M^{\prime}$.

\section{Theorem 2.3.2}

Suppose $\mathrm{M}$ is a self-dual 2-connected matroid with self-dual permutation $\delta$ and let $e_{1} \in M$. Let $\left\{e_{1}, e_{2}, \ldots \ldots e_{k}\right\}$ be the orbit of $e_{1}$ under $\delta$. Suppose one of the following:

1) $\mathrm{k}$ is even and $M_{0}$ is a 3-connected matroid or a cycle and $\delta_{0}$ is a matroid automorphism of $M_{0}$ fixing an edge $e_{0}$.

2) $\mathrm{K}$ is odd and $M_{0}$ is a 3-connected self-dual matroid with self-dual permutation $\delta_{0}$ fixing an edge $e_{0}$. For $\mathrm{i}=1,2, \ldots, \mathrm{k}$ set $M_{2 i+1}=M_{0}$ and $M_{2 i}=M_{0} *$. Let $M^{\prime}$ be the matroid obtained from $\mathrm{M}$ by 2-sums with the matroids $M_{i}$, amalgamating $e_{0}$ or $e_{0} *$ in $M_{i}$ with $e_{i}$.

Let $\delta^{\prime}$ be defined by $\delta^{\prime}$ (e) for e $\in M-\left\{e_{1}, e_{2}, \ldots . ., e_{k}\right\}, \delta^{\prime}: M_{i}-e_{0} \rightarrow M_{i+1}-e_{0}$ is induced by $*$ for $\mathrm{i}=1$, $2, \ldots ., \mathrm{k}$ and $\delta^{\prime}=\delta_{0}: M_{k} \rightarrow M_{1}$. Then $M^{\prime}$ is a 2-connected self-dual matroid with self-dual permutation $\delta^{\prime}$. Moreover, every 2-connected self-dual matroid and its self-duality is obtained in this manner.

Proof

The fact that this construction gives a 2-connected self-dual matroid follows at once, since to check if $\delta^{\prime}$ is a self-duality, it sufficient to check that $\left(\delta^{\prime}\right)_{\alpha}$ sends cycles to co-cycles on each 3-block. The fact that 
$M_{0}$ must be self-dual if $\mathrm{K}$ is odd follows by considering that $\delta^{1 k}$ is a self-duality and maps $M_{0}=M_{1}$ onto itself.

To see that all self-dualities arise this way, let $\delta^{\prime}: M^{\prime} \rightarrow M^{\prime}$ be a self-duality, let $\alpha$ be a pendant node of T, and set $M_{0}=M_{\alpha}$. Let $\mathrm{M}$ be the self-dual matroid that results from removing from $T\left(M^{\prime}\right)$ the $\mathrm{K}$ nodes corresponding to the orbit of the node $\alpha . \delta^{\prime}$ induces $\delta: \mathrm{M} \rightarrow \mathrm{M}$. Then the desired $\delta_{0}$ is $\left(\delta^{k}\right)_{\alpha}$.

\subsection{Ribbon Graphs}

\section{A Characterisation Of Partial Dual Graphs}

S. Chmutov recently introduced the concept of the partial dual $G^{A}$ of a ribbon graph G. Partial duality generalizes the natural dual (or Euler- Poincare dual or geometric dual) of a ribbon graph by forming the dual of $\mathrm{G}$ with respect to a subset of its edges A. In contrast with natural duality, where the topologies of $\mathrm{G}$ and $\mathrm{G}^{*}$ are similar, the topology of a partial dual $\mathrm{G}^{*}$ can be very different from the topology of $\mathrm{G}$.

For Example, Although a ribbon graph and its natural dual always have the same genus, a ribbon graph and a partial dual need not.

\section{Definition 3.1.1}

A ribbon graph $G=(v(G), \varepsilon(G))$ is (possibly non-orientable) surface with boundary represent as the union of two sets of topological discs: a set $v(\mathrm{G})$ of vertices, and set of edges $\varepsilon(\mathrm{G})$ such that

(i) The vertices and edges intersect in disjoint line segment.

(ii) Each such line segment lies on the boundary of precisely one vertex and precisely one edge;

(iii) Every edge contains exactly two such line segments.

It will be convenient to use a description of a ribbon graph $\mathrm{G}$ as a spanning sub- ribbon graph equipped with a set of colored arrows that record where the missing edges.

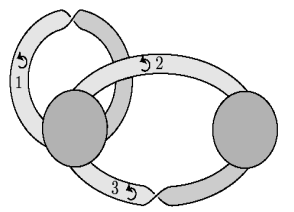

(i)

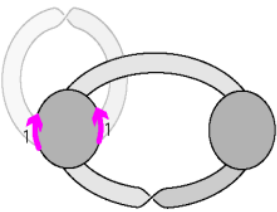

(ii)

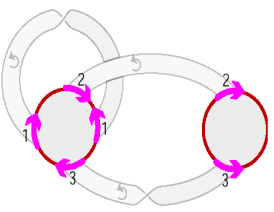

(iii)

\section{Definition 3.1.2}

Fig.: 3.1 Realizations of a ribbon graph.

An arrow marked ribbon graph $\vec{G}$ consists of a ribbon graph $\mathrm{G}$ equipped with a collection of colored arrows, called marking arrows, on the boundaries of its vertices. The marking arrows are such that no marking arrow meets an edge of the ribbon graph, and there are exactly two marking arrows of each other.

\section{Illustration}

A ribbon graph can be obtained from an arrow-marked ribbon graph by adding edges in a way prescribed by the marking arrows, thus take a disc and orient its boundary arbitrarily. Add this disc to the ribbon graph by choosing two non-interesting arcs on the boundary of the disc and two marking arrows on the same color, and then identifying the arcs with the marking arrows according to the orientation of the arrow. The disc that has been added forms an edge of a new ribbon graph.

This process is illustrated in Fig,:3.2, and an example of an arrow -marked ribbon graph and the ribbon graph it describes in Fig.:3.1 (i) and (ii).

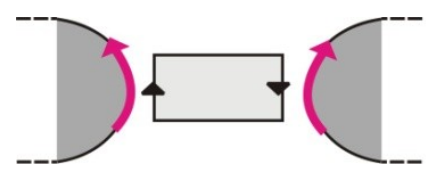

Result

An arrow-marked ribbon graph describes a ribbon graph. Conversely, every ribbon graph can be described as an arrow-marked spanning sub-ribbon graph.

Proof

Suppose that $\mathrm{G}$ is a ribbon graph and $\mathrm{B} \subset \varepsilon(\mathrm{G})$.

To describe $\mathrm{G}$ as an arrow-marked ribbon graph, $\overrightarrow{G \backslash B}$ start by arbitrarily orienting each edge in $\mathrm{B}$. This induces an orientation on the boundary of each edge in $\mathrm{B}$. To construct the marking arrows for each e $\in \mathrm{B}$, place 
an arrow on each of the two arcs where e meets vertices of G, the direction of this arrow should follow the orientation of the boundary e, color the two arrows with e and delete the edge e. This gives a marked ribbon graph $\overrightarrow{G \backslash B}$. Moreover, the original ribbon graph $\mathrm{G}$ can be recovered from $\overrightarrow{G \backslash B}$ by adding edges of $\overrightarrow{G \backslash B}$ as prescribed by the marking arrows.

Notice that, if $\mathrm{G}$ is a ribbon graph and $\mathrm{H}$ is any spanning sub-ribbon graph, then there is an arrow marked ribbon graph of $\vec{H}$ which describes $\mathrm{G}$.

Definition 3.1.3

An arrow presentation of a ribbon graph consists of a set of oriented (topological) circles (called cycles) that are marked with colored arrows called marking arrows, such that there are exactly two marking arrows of each color.

\section{Example}

An example of a ribbon graph and its arrow presentation is given in below figure.
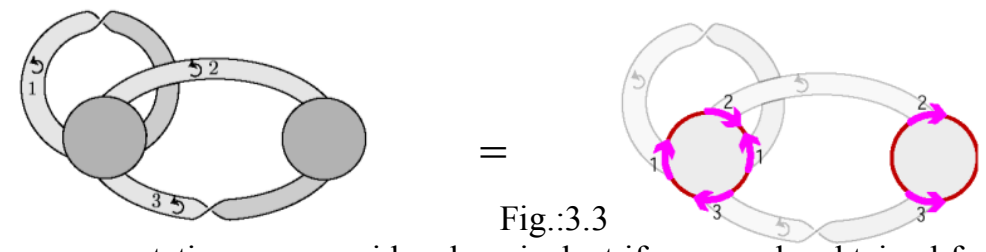

Two arrow presentations are considered equivalent if one can be obtained from the other by reversing pairs of marking arrows of the same color.

\subsection{Partial Duality}

Partial duality is a generalization of the natural dual of a ribbon graph. A key feature of partial duality is that it provides a way extend the well known relation $T(G ; x, y)=T\left(G^{*} ; y, x\right)$, relating the Tutte polynomial of a planar graph and its dual, to the weighted ribbon graph polynomial.

Although the construction of the partial dual $G^{A}$ of $g$ is perhaps a little lengthy to write down, in practice the formation of the partial dual is a straightforward process.

\section{Definition 3.2.1}

Let $\mathrm{G}$ be a ribbon graph and $A \subseteq \varepsilon(G)$. The partial dual $G^{A}$ of $\mathrm{G}$ along A is defined below. (Step P1.) :

Give every edge in $\varepsilon(G)$ orientation (this need not extend to an orientation of the whole ribbon graph). Construct a set of marked, oriented, disjoint paths on the boundary of the edges of $G$ in the following way:

(1) If $\mathrm{e} \notin \mathrm{A}$ then the intersection of the edge $e$ with distinct vertices (or vertex if e is a loop) defines two paths. Mark each of these paths with an arrow which points in the direction of the orientation of the boundary of the edge. Color both of these marks with $e$.

(2) If $\mathrm{e} \in \mathrm{A}$ then the two sides of $e$ which do not meet the vertices define the two paths.

Mark each of these paths with an arrow which points in the direction of the orientation of the boundary (Step P2):

of the edge. Color both of these marks with $e$.

Construct a set of closed curves on the boundary of $\mathrm{G} \backslash A^{c}$ by joining the marked paths constructed above by connecting them along the boundaries of $\mathrm{G} \backslash A^{c}$ in the natural way.

(Step P3):

This defines a collection of non-interesting, closed curves on the boundary of $\mathrm{G} \backslash A^{c}$ which are marked with colored, oriented arrows. This is precisely an arrow presentation of a ribbon graph. The corresponding ribbon graph is the partial dual of $\mathrm{G}^{\mathrm{A}}$.

The construction is shown locally at an edge e in Fig.:2.4

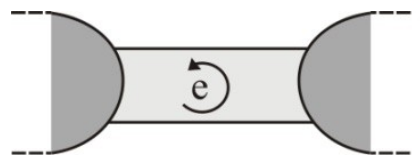

An untwisted edge e

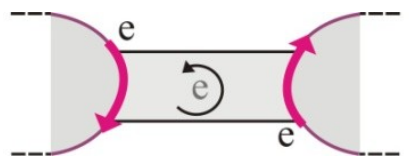

If e $\notin A$

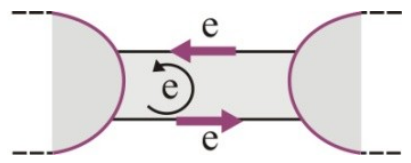

If $\mathrm{e} \in \mathrm{A}$ 


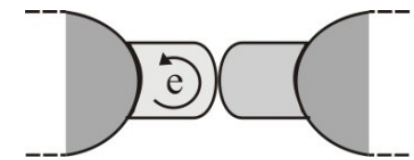

A twisted edge e

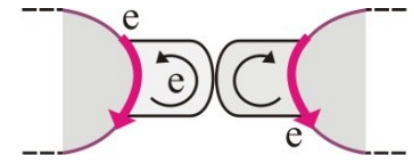

If e $\notin A$

Fig.:3.4 Forming paths in the partial dual.

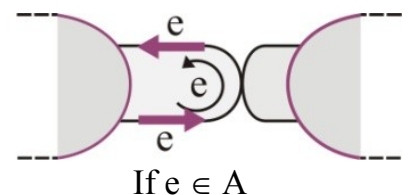

If $\mathrm{e} \in \mathrm{A}$

\section{Example 1}

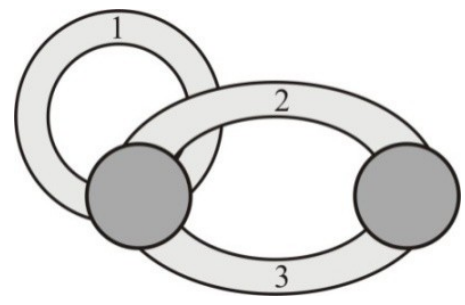

G with $A=\{2,3\}$
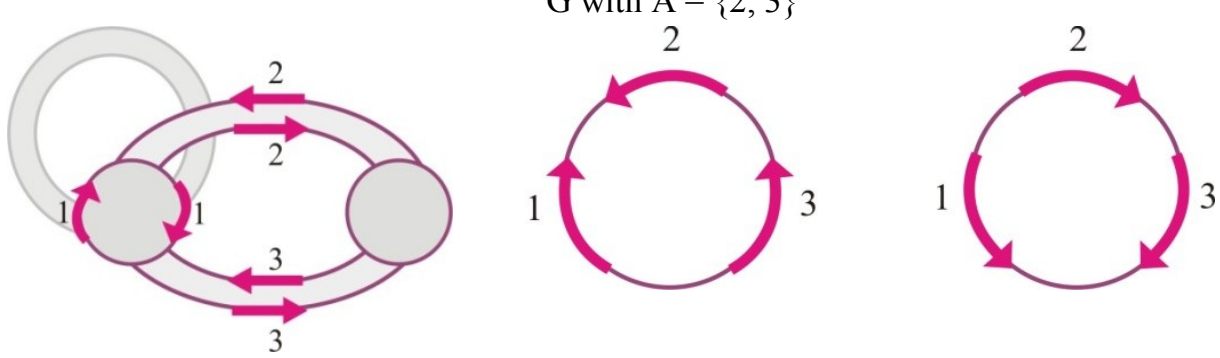

Steps P1 and P2
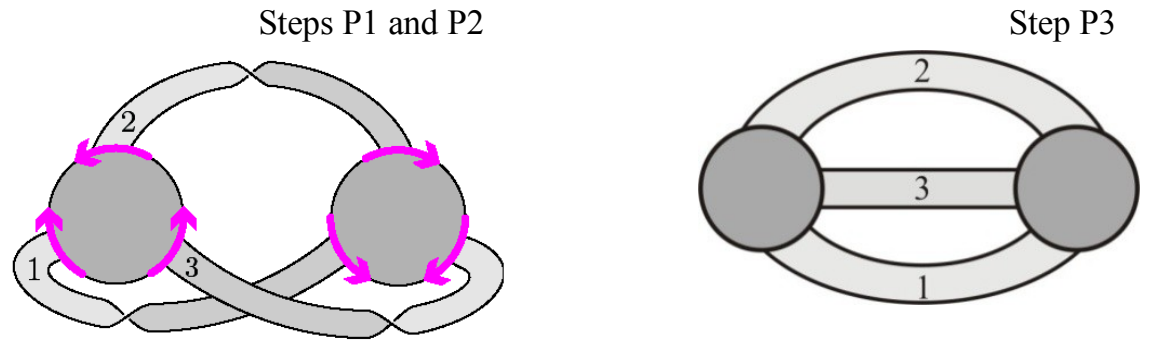

$\mathrm{G}^{\mathrm{A}}$

Redrawing $\mathrm{G}^{\mathrm{A}}$

Fig.:3.5

\section{Example 2}

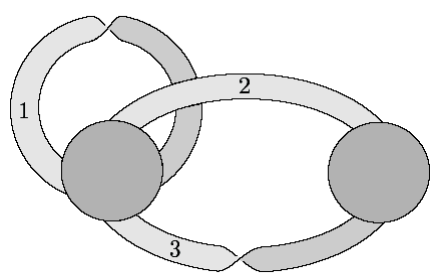

$\mathrm{G}$ with $\mathrm{A}=\{2,3\}$

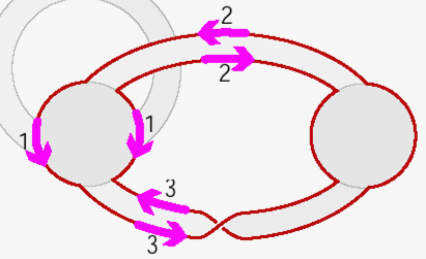

Steps P1 and P2

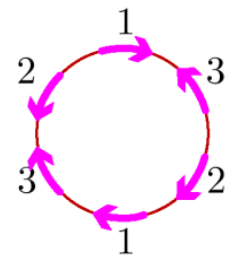

Step P3 


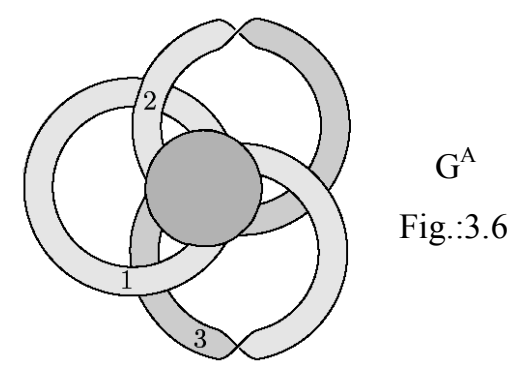

\section{Natural Duality}

Let $\mathrm{G}=(v(G), \varepsilon(G))$ be a ribbon graph. We can regard $\mathrm{G}$ as a punctured surface. By filling in the punctures using a set of discs denoted $v\left(G^{*}\right)$.We obtain a surface without boundary $\sum$. The natural dual (or Euler-Poincare dual) of $\mathrm{G}$ is the ribbon graph $\mathrm{G}^{*}=\left(\nu\left(G^{*}\right), \varepsilon(G)\right)$.

\subsection{Partial Dual Embedding}

A dual embedding $\{\mathrm{G}, \mathrm{H}, \Sigma\}$ of $\mathrm{G}$ and $\mathrm{H}$ into a surface $\sum$ to be an embedding of $\mathrm{G}$ in a surface without boundary $\sum$ which has the property that $\mathrm{H}=\sum \backslash \mathrm{v}(\mathrm{G})$

Note that a dual embedding is independent of the order of the ribbon graphs $\mathrm{G}$ and $\mathrm{H}$ (i.e. the dual embeddings $\{\mathrm{G}, \mathrm{H}, \Sigma\}$ and $\{\mathrm{H}, \mathrm{G}, \Sigma\}$ are equivalent).

\section{Definition 3.3.1}

A set $\{\mathrm{G}, \mathrm{H}, \Sigma, \mathrm{M}\}$ is a partial dual embedding of ribbon graph $\mathrm{G}$ and $\mathrm{H}$ if

i) $\{\mathrm{G}, \mathrm{H}, \Sigma\}$ is a dual embedding;

ii) $\mathrm{M}$ is a set of disjoint colored arrows marked on the boundaries of the embedded vertices in $v(G) \cap v(H) \subset \Sigma$ with the property that there are exactly two arrows of each color.

\section{Theorem 3.3.1}

Let $\mathrm{G}$ and $\mathrm{H}$ be ribbon graphs. Then $\mathrm{G}$ and $\mathrm{H}$ are partial duals if and only if there exists a partial dual embedding $\{\tilde{G}, \tilde{H}, \Sigma, \mathrm{M}\}$ with the property that $\Sigma \backslash \nu(\tilde{H}) \cup \mathrm{M}$ is an arrow-marked ribbon graph describing G, and $\Sigma \backslash v(\tilde{G}) \cup \mathrm{M}$ is an arrow-marked ribbon graph describing $\mathrm{H}$.

\section{Proof}

First suppose that $\mathrm{G}$ and $\mathrm{H}$ are partial duals. Then there exists a set of edges $\mathrm{A} \subseteq \mathcal{E}(\mathrm{G})$ such that $G^{A}=\mathrm{H}$. Then $\mathrm{G}$ can described as an arrow-marked ribbon graph $\overrightarrow{G \backslash A^{c}}$, where $A^{c}=\varepsilon$ (A)\A. Let $\Sigma$ be the surface obtained from $G \backslash A^{c}$ by filling in the punctures. Then $\left\{G \backslash A^{c},\left(G \backslash A^{c}\right)^{*}, \Sigma\right\}$ forms a natural dual embedding. The arrow markings on $\overrightarrow{G \backslash A^{c}}$ induce a set of colored arrows on $v\left(G \backslash A^{c} \cap v\left(\left(G \backslash A^{c}\right)^{*}\right)\right.$ with the property that there are exactly two arrows of each color. Denote this induced set of colored arrows by $\mathrm{M}$.

Then $\left\{G \backslash A^{c},\left(G \backslash A^{c}\right)^{*}, \Sigma, \mathrm{M}\right\}$ is a partial dual embedding. Moreover, $\Sigma \backslash v\left(\left(G \backslash A^{c}\right)^{*}\right)$ describes $\mathrm{G}$ by construction, and $\Sigma \backslash \nu\left(\left(G \backslash A^{c}\right)\right)$ clearly describes $G^{A}=\mathrm{H}$ if we use the construction of partial duality from the lemma,

"Let $\mathrm{G}$ be a ribbon graph, $A \subset \varepsilon(G)$ and $A^{c}=\varepsilon(\mathrm{A}) \backslash \mathrm{A}$. Then the following construction gives $G^{A}$

(Step $P 1^{\prime}$ ): Present $\mathrm{G}$ as the arrow-marked ribbon graph $\overrightarrow{G \backslash A^{c}}$.

(Step $P 2^{\prime}$ ): Take the natural dual of $G \backslash A^{c}$. The marking arrows on $\overrightarrow{G \backslash A^{c}}$ induce marking arrows on $\left(G \backslash A^{c}\right)^{*}$.

(Step $\left.P 3^{\prime}\right): G^{A}$ is the ribbon graph corresponding to the arrow-marked ribbon graph $\overline{\left(G \backslash A^{c}\right)^{*}}$ ".

Conversely, suppose that $\{\tilde{G}, \tilde{H}, \Sigma, \mathrm{M}\}$ is a partial dual embedding with the property that $\Sigma \backslash \mathcal{V}(\tilde{H}) \cup \mathrm{M}$ is an arrow-marked ribbon graph describing $\mathrm{G}$, and $\Sigma \backslash v(\tilde{G}) \cup \mathrm{M}$ is an arrow marked ribbon graph describing H. Then $\tilde{G}$ and $\tilde{H}$ are precisely the naturally dual marked ribbon graphs described in step $P 2^{\prime}$ of the construction of partial dual. Here A is the set of edges of $\mathrm{G}$ that are also in $G^{\prime}$. 


\subsection{Partial Duality for Graphs}

\section{Definition 3.4.1}

If $\mathrm{G}=(v(G), \varepsilon(G))$ is a ribbon graph then we can construct a graph $\mathrm{G}=(\nu(G), \varepsilon(G))$ form $\mathrm{G}$ by replacing each edge of $\mathrm{G}$ with a line, and then contracting the vertices of $\mathrm{G}$ into points, such a graph $\mathrm{G}$ is called the core of G.

Notice that there is a natural correspondence between the edges of a ribbon graph and its core, and the vertices of a ribbon graph and its core.

\section{Definition 3.4.2}

We say that two graphs are partial duals if they are cores of partially dual ribbon graphs.

Let $\mathrm{G}$ be a ribbon graph and $A \subseteq \varepsilon(\mathrm{G})$. By the notation $G^{A}$ we mean that $G^{A}$ is the core of $G^{A}$ where $\mathrm{G}$ is the core of $\mathrm{G}$ and $\mathrm{A}$ is the edge set of $\mathrm{G}$ that corresponds with $\mathrm{A}$.

We have seen that partially dual ribbon graphs can be characterized by the existence of an appropriate partially dual embedding. A corresponding result holds partial dual graphs. To describe the corresponding result, we make the following definition.

\section{Definition 3.4.3}

A partial dual embedding of graphs is a set $\{\tilde{G}, \tilde{H}, \Sigma, \mathrm{E}\}$, Where $\Sigma$ is a surface without boundary $\tilde{G}, \tilde{H} \subset \Sigma$ are embedded graphs and $\mathrm{E}$ is a set of colored edges that are embedded in $\Sigma$ such that

(1) Only the ends of each embedded edge in $\mathrm{E}$ meet $\tilde{G} \cup \tilde{H} \subset \Sigma$;

(2) $\{\tilde{G}, \tilde{H}, \Sigma\}$ is dual embedding;

(3) Each edge in $\mathrm{E}$ is incident to one vertex in $v(\tilde{G})$ and one vertex in $v(\tilde{H})$;

(4) There are exactly two edges of each color in E.

\section{Example}

An example of partial dual embedding

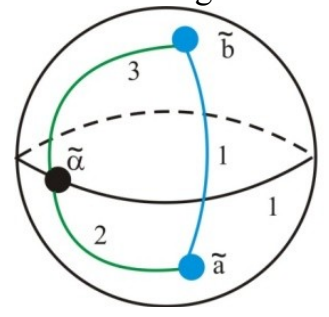

Fig.: 3.7

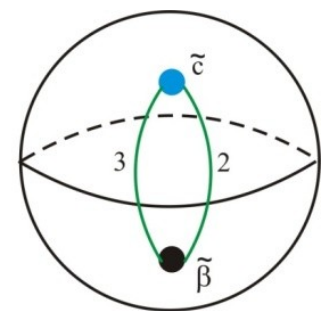

Where $\Sigma$ is the disjoint union of two spheres, $\tilde{G}_{1}=(\{\tilde{\alpha}, \tilde{\beta}\},\{1\})$ and $\tilde{G}_{2}=(\{\tilde{a}, \tilde{b}, \tilde{c}\},\{1\})$.

Following the recipe in the theorem we recover the graphs.

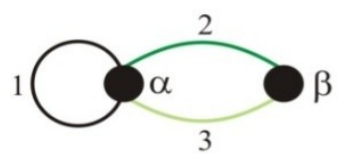

G

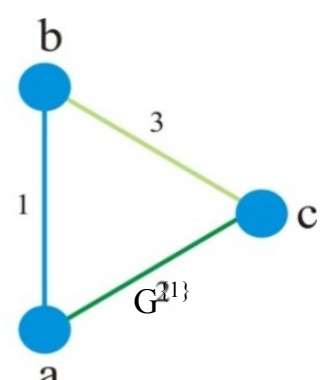

a

These graphs are indeed partial duals as they are cores of the following graphs respectively.
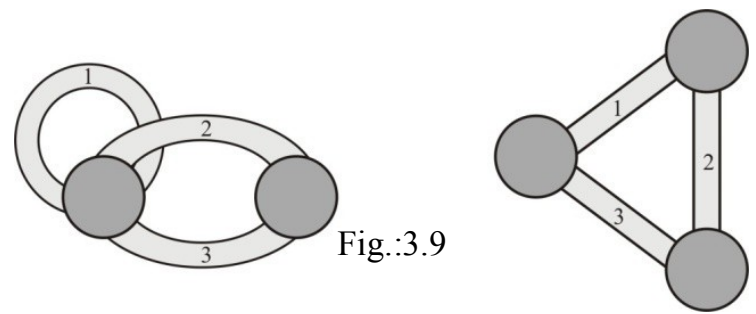


\section{Theorem 3.4.1}

Two graphs $G_{1}$ and $G_{2}$ are partial duals if and only if there exists a partial dual embedding $\left\{\tilde{G}_{1}, \tilde{G}_{2}, \Sigma, \mathrm{E}\right\}$ such that for each i, $G_{i}$ is obtained from $\tilde{G}_{i}$ by adding an edge between the vertices of $\tilde{G}_{i}$, that are incident with the two edges in $\mathrm{E}$ that have the same color, for each color.

Proof

First suppose that $G_{1}$ and $G_{2}$ are partial duals, so $G_{1}$ and $G_{2}$ are the cores of partially dual ribbon graphs. Then by theorem, there exists a partial dual embedding $\left\{\tilde{G}_{1}, \tilde{G}_{2}, \Sigma, \mathrm{M}\right\}$ such that $\Sigma \backslash v\left(\tilde{G}_{2}\right) \cup \mathrm{M}$ is an arrow-marked ribbon graph describing $G_{1}=\Sigma \backslash v\left(\tilde{G}_{1}\right) \cup \mathrm{M}$ is an arrow-marked ribbon graph describing $G_{2}: G_{1}$ is the core of $G_{1}$ and $G_{2}$ is the core of $G_{2}$.

A partial dual embedding of graphs $\left\{\tilde{G}_{1}, \tilde{G}_{2}, \Sigma\right.$, E $\}$ can be constructed from $\left\{\tilde{G}_{1}, \tilde{G}_{2}, \Sigma, \mathrm{M}\right\}$ in the following way: Let $\tilde{G}_{1}$ be the canonically embedded core of $\tilde{G}_{1}$ and $\tilde{G}_{2}$ let be the canonically embedded core of $\tilde{G}_{2}$. Each arrow on $\Sigma$ meets exactly two vertices of $\tilde{G}_{1} \cup \tilde{G}_{2}$. For each arrow, add an embedded edge between the two corresponding vertices of the graph $\tilde{G}_{1} \cup \tilde{G}_{2} \subset \Sigma$ which passes through this arrow. Color the edge with the color of the arrow that it passes through. The set of edges added in this way forms $\mathrm{E}$.

Now to show that $\left[\tilde{G}_{1}, \tilde{G}_{2}, \Sigma, E\right\}$ is indeed a partial dual embedding of graphs and the graphs $G_{1}$ and $G_{2}$ can be recovered from the partial dual embedding in the way described by the theorem.

To see that $\left[\tilde{G}_{1}, \tilde{G}_{2}, \Sigma, \mathrm{E}\right\}$ is a partial dual embedding, first note that by construction $\tilde{G}_{1}, \tilde{G}_{2}$ and E are all embedded in $\Sigma$, and that only the ends of the edges in E meet $\tilde{G}_{1}$ or $\tilde{G}_{2} \cdot\left\{\tilde{G}_{1}, \tilde{G}_{2}, \Sigma\right\}$ is a dual embedding.

Since each arrow in $\mathrm{M}$ meets one vertex in $V\left(G_{1}\right)$ and one vertex in $V\left(G_{2}\right)$, each edge in $\mathrm{E}$ is incident to vertex in $V\left(\tilde{G}_{1}\right)$ and one vertex in $V\left(\tilde{G}_{2}\right)$. The coloring requirement follows since there are exactly two edges of each color in $M$ and the edge colorings of $E$ are induced from $M$.

Finally, $G_{i}$ can be recovered from $\tilde{G}_{i} \cup \mathrm{M}$ by adding edges between the marking arrows of the same color. Therefore, if $\mathrm{u}$ and $\mathrm{v}$ are vertices of $\tilde{G}_{i}$ which are marked with an arrow of the same color and $\mathrm{u}$ and $\mathrm{v}$ are vertices of $\tilde{G}_{i}$ which are marked with an arrow of the same color and $\mathrm{u}$ and $\mathrm{v}$ are the corresponding vertices of $\tilde{G}_{i}$, then to construct the core of $G_{i}$ we need to add an edge between u and v.

But since $\mathrm{u}$ and $\mathrm{v}$ are each incident with the edges in $\mathrm{E}$ of the same color we need to add an edge between the vertices of $\tilde{G}_{i}$ that are incident with the two edges in E of the same color. This is exactly the construction described in the statement of the theorem. Using this for each color gives $G_{i}$, completing the proof of necessity.

Conversely, suppose that $\left\{\tilde{G}_{1}, \tilde{G}_{2}, \Sigma, \mathrm{E}\right\}$ is a partial dual embedding and that $G_{1}$ and $G_{2}$ are obtained as described in the statement of the theorem. Construct a partial dual embedding $\left\{\tilde{G}_{1}, \tilde{G}_{2}, \Sigma, \mathrm{M}\right\}$ of ribbon graph in the following way take a small neighborhood in $\Sigma$ of the embedded graph $\tilde{G}_{1}$ to form $\tilde{G}_{1}$; let $G_{2}=\left(\Sigma \backslash \tilde{G}_{1}, \varepsilon\left(\tilde{G}_{1}\right)\right)$ wherever an edge in E meets a boundary of vertices add an arrow pointing in an arbitrary direction which is colored by the color of the edge in E. M is the set of such colored arrows.

To see that $\left\{\tilde{G}_{1}, \tilde{G}_{2}, \Sigma, M\right\}$ is a partial dual embedding, note that $\left\{\tilde{G}_{1}, \tilde{G}_{2}, \Sigma\right\}$ is a dual embedding since $\left\{\tilde{G}_{1}, \tilde{G}_{2}, \Sigma\right\}$ is, and that there exactly two arrows of each color since there are exactly two edges of each color in $\mathrm{E}$.

Let $G_{i}$ denote the ribbon graph described by the arrow-marked ribbon graph $\tilde{G}_{i} \cup \mathrm{M}$. Then $G_{i}$ is the core of $G_{i}$ (since whenever an edge is added between two vertices of $\tilde{G}_{i}$ in the formation of $G_{i}$, an edge is 
added between the corresponding vertices of $\tilde{G}_{i}$ in the formation of $G_{i}$ ). Finally, $G_{1}$ and $G_{2}$ are partial dual graphs since, by Theorem: $G_{1}$ and $G_{2}$ are partial dual ribbon graphs.

\section{Conclusion}

This dissertation deals with self-dual graphs and characterization of partial dual graphs. In this paper first we studied about Forms of self-dual graphs and comparisons of forms of self dual graphs are discussed. And finally Characterization of partial dual graphs are studied.

\section{References}

[1] Brigitte Servatius and Herman Servatius Self-Dual Graphs, Discrete Math., 1996.

[2] Iain Moffatt, A Characterization of Partially Dual Graphs, Google.

[3] J. Edmonds, On the Surface Duality of linear graphs, J. Res.Nat.Bur.Standards sect. B 69B (1965) 121-123

[4] H.Whitney, Non-separable and Planar Graphs

[5] W.T.Tutte, Connectivity in Graphs (Univ. Toronto press, Toronto, 1966).

[6] W.T.Tutte, Connectivity in matroids, canad. J. Math.18(1966) 1301-1324

[7] H.Whitney, 2-isomorphic graphs, Amer. J. Math. 55(1933) 245-254.

[8] Sergei Chmutov, Generalized duality for graphs on surfaces and the signed Bollob_as-Riordan polynomial, J. Combin. Theory Ser. B, 99 (3) (2009) 